Kragujevac Journal of Mathematics

Volume 45(4) (2021), Pages 615-622.

\title{
ON BERNSTEIN-TYPE INEQUALITIES FOR RATIONAL FUNCTIONS WITH PRESCRIBED POLES
}

\author{
ABDULLAH MIR ${ }^{1}$
}

\begin{abstract}
In this paper, we shall use a parameter $\beta$ and obtain some Bernsteintype inequalities for rational functions with prescribed poles which generalize the results of Qasim and Liman and Li, Mohapatra and Rodriguez and others.
\end{abstract}

\section{INTRODUCTION}

Let $\mathbb{P}_{n}$ denote the class of all complex polynomials of degree at most $n$. If $P \in \mathbb{P}_{n}$, then concerning the estimate of $\left|P^{\prime}(z)\right|$ on $|z|=1$, we have

$$
\left|P^{\prime}(z)\right| \leq n \sup _{|z|=1}|P(z)| \text {. }
$$

Inequality (1.1) is a famous result due to Bernstein [2], who proved it in 1912. Later, in 1969 (see [10]), Malik improved the above inequality (1.1) and established that if $P \in \mathbb{P}_{n}$, then for $|z|=1$, we have

$$
\left|P^{\prime}(z)\right|+\left|Q^{\prime}(z)\right| \leq n \sup _{|z|=1}|P(z)|,
$$

where $Q(z)=z^{n} \overline{P\left(\frac{1}{\bar{z}}\right)}$.

It is worth mentioning that equality holds in (1.1) if and only if $P(z)$ has all its zeros at the origin, so it is natural to seek improvements under appropriate assumption on the zeros of $P(z)$. If we restrict ourselves to the class of polynomials

Key words and phrases. Rational function, polynomial, poles, zeros.

2010 Mathematics Subject Classification. Primary: 30A10. Secondary: 30C10, 30D15.

DOI 10.46793/KgJMat2104.615M

Received: September 04, 2018.

Accepted: April 04, 2019. 
$P(z)$ having no zeros in $|z|<1$, then (1.1) can be replaced by

$$
\sup _{|z|=1}\left|P^{\prime}(z)\right| \leq \frac{n}{2} \sup _{|z|=1}|P(z)|
$$

whereas if $P(z)$ has no zeros in $|z|>1$, then

$$
\sup _{|z|=1}\left|P^{\prime}(z)\right| \geq \frac{n}{2} \sup _{|z|=1}|P(z)| .
$$

Inequality (1.3) was conjectured by Erdös and later verified by Lax [9], whereas inequality (1.4) is due to Turán [12]. Li, Mohapatra and Rodriguez [14] gave a new perspective to the above inequalities and extended them to rational functions with prescribed poles. Essentially, in the inequalities referred to, they replaced the polynomial $P(z)$ by a rational function $r(z)$ with prescribed poles $a_{1}, a_{2}, \ldots, a_{n}$ and $z^{n}$ by a Blaschke product $B(z)$. Before proceeding towards their results, let us introduce the set of rational functions involved.

For $a_{j} \in \mathbb{C}$ with $j=1,2, \ldots, n$, let

$$
W(z):=\prod_{j=1}^{n}\left(z-a_{j}\right)
$$

and let

$$
B(z):=\prod_{j=1}^{n}\left(\frac{1-\bar{a}_{j} z}{z-a_{j}}\right), \quad \mathcal{R}_{n}:=\mathcal{R}_{n}\left(a_{1}, a_{2}, \ldots, a_{n}\right)=\left\{\frac{P(z)}{W(z)}: P \in \mathbb{P}_{n}\right\} .
$$

Then $\mathcal{R}_{n}$ is the set of rational functions with poles $a_{1}, a_{2}, \ldots, a_{n}$ at most and with finite limit at $\infty$. Note that $B(z) \in \mathcal{R}_{n}$ and $|B(z)|=1$ for $|z|=1$. For $r(z)=\frac{P(z)}{W(z)} \in \mathcal{R}_{n}$, the conjugate transpose $r^{*}$ of $r$ is defined by $r^{*}(z)=B(z) \overline{r\left(\frac{1}{\bar{z}}\right)}$. The rational function $r \in \mathcal{R}_{n}$ is called self-inversive if $r^{*}(z)=\lambda r(z)$ for some $\lambda$ with $|\lambda|=1$.

As an extension of (1.2) to rational functions, Li, Mohapatra and Rodriguez [14, Theorem 2] showed that if $r \in \mathcal{R}_{n}$, then

$$
\left|r^{\prime}(z)\right|+\left|\left(r^{*}(z)\right)^{\prime}\right| \leq\left|B^{\prime}(z)\right| \sup _{|z|=1}|r(z)|, \quad \text { for }|z|=1 .
$$

Equality holds in (1.5) for $r(z)=\alpha B(z)$ with $|\alpha|=1$.

For $r \in \mathcal{R}_{n}$ to be self-inversive, Li, Mohapatra and Rodriguez [14, Corollary 4] proved that

$$
\left|r^{\prime}(z)\right| \leq \frac{\left|B^{\prime}(z)\right|}{2} \sup _{|z|=1}|r(z)| .
$$

In the same paper, Li, Mohapatra and Rodriguez [14] showed that inequality (1.6) also holds for rational functions $r \in \mathcal{R}_{n}$ having no zeros in $|z|<1$ with prescribed poles. The latest development of further results along this line can be found in the monographs and papers $[3-5,7,8,11]$. 
More recently, Qasim and Liman [6] proved several results by considering a specialized class of rational functions $r(t(z))$, defined by

$$
(r \circ t)(z)=r(t(z)):=\frac{P(t(z))}{W(t(z))},
$$

where $t(z)$ is a polynomial of degree $m$ and $r \in \mathcal{R}_{n}$, so that $r(t(z)) \in \mathcal{R}_{m n}$, and

$$
W(t(z))=\prod_{j=1}^{m n}\left(z-a_{j}\right) .
$$

Also the Blaschke product is given by

$$
B(z)=\frac{(W(t(z)))^{*}}{W(t(z))}=\frac{z^{m n} \overline{W\left(t\left(\frac{1}{\bar{z}}\right)\right)}}{W(t(z))}=\prod_{j=1}^{m n}\left(\frac{1-\bar{a}_{j} z}{z-a_{j}}\right) .
$$

Assume that the $m n$ poles of $r(t(z))$ are denoted by $a_{j}, j=1,2, \ldots, m n$, and $\left|a_{j}\right|>1$. They proved the following Bernstein-type inequality for rational functions $r(t(z)) \in \mathcal{R}_{m n}$ with restricted zeros.

Theorem 1.1. If $r(t(z)) \in \mathcal{R}_{m n}$ and all the mn zeros of $r(t(z))$ lie in $|z| \geq 1$, then for $|z|=1$

$$
\left|r^{\prime}(t(z))\right| \leq \frac{\left|B^{\prime}(z)\right|}{2 m \mu} \sup _{|z|=1}|r(t(z))|
$$

where $t(z)$ has all its zeros in $|z| \leq 1$ and $\mu=\inf _{|z|=1}|t(z)|$.

\section{LEMMAS}

For the proofs of our theorems we need the following lemmas.

Lemma 2.1. If $r \in \mathcal{R}_{n}$ has $n$ zeros all lie in $|z| \leq 1$, then

$$
\left|r^{\prime}(z)\right| \geq \frac{1}{2}\left|B^{\prime}(z)\right||r(z)|, \quad \text { for }|z|=1 .
$$

The above lemma is due to Li, Mohapatra and Rodriguez [14].

Lemma 2.2. Let $A$ and $B$ be any two complex numbers, then

(i) if $|A| \geq|B|$ and $B \neq 0$, then $A \neq \delta B$ for all complex numbers $\delta$ satisfying $|\delta|<1$;

(ii) conversely, if $A \neq \delta B$ for all complex numbers $\delta$ satisfying $|\delta|<1$, then $|A| \geq|B|$.

The above lemma is due to $\mathrm{Li}[13]$. 
Lemma 2.3. If $r(t(z)), s(t(z)) \in \mathcal{R}_{m n}$ and all the $m n$ zeros of $s(t(z))$ lie in $|z| \leq 1$ and $|r(t(z))| \leq|s(t(z))|$ for $|z|=1$. Then for every $\beta \in \mathbb{C}$, with $|\beta| \leq 1$ and $|z|=1$, we have

$$
\left|B(z) r^{\prime}(t(z)) t^{\prime}(z)+\frac{\beta}{2} B^{\prime}(z) r(t(z))\right| \leq\left|B(z) s^{\prime}(t(z)) t^{\prime}(z)+\frac{\beta}{2} B^{\prime}(z) s(t(z))\right| .
$$

The result is sharp and equality holds in (2.1) for $r(t(z))=\alpha s(t(z))$, with $|\alpha|=1$.

Proof. The proof of this lemma is identical to the proof of Theorem 3.2 of $\mathrm{Li}$ [13], but for the sake of completeness we give the brief outlines of its proof. First assume that no zero of $s(t(z))$ are on the unit circle $|z|=1$ and therefore, all the $m n$ zeros of $s(t(z))$ are in $|z|<1$. By Rouche's theorem, the rational function $\lambda r(t(z))+s(t(z))$ has all its zeros in $|z|<1$ for $|\lambda|<1$ and has no poles in $|z| \leq 1$. On applying Lemma 2.1 to $\lambda r(t(z))+s(t(z))$, we get on $|z|=1$

$$
2|B(z)|\left|\lambda(r(t(z)))^{\prime}+(s(t(z)))^{\prime}\right| \geq\left|B^{\prime}(z)\right||\lambda r(t(z))+s(t(z))| .
$$

Now, note that $B^{\prime}(z) \neq 0$ (e.g. see formula (14) in [14]). So, the right hand side of (2.2) is non zero. Thus, by using $(i)$ of Lemma 2.2 , we have for all $\beta \in \mathbb{C}$, with $|\beta|<1$,

$$
2 B(z)\left(\lambda r^{\prime}(t(z)) t^{\prime}(z)+s^{\prime}(t(z)) t^{\prime}(z)\right) \neq-\beta B^{\prime}(z)(\lambda r(t(z))+s(t(z))),
$$

for $|z|=1$. Equivalently, for $|z|=1$,

$$
\lambda\left(2 B(z) r^{\prime}(t(z)) t^{\prime}(z)+\beta B^{\prime}(z) r(t(z))\right) \neq-\left(2 B(z) s^{\prime}(t(z)) t^{\prime}(z)+\beta B^{\prime}(z) s(t(z))\right),
$$

for $|\lambda|<1$ and $|\beta|<1$. Using (ii) of Lemma 2.2, we have

$$
\left|2 B(z) r^{\prime}(t(z)) t^{\prime}(z)+\beta B^{\prime}(z) r(t(z))\right| \leq\left|2 B(z) s^{\prime}(t(z)) t^{\prime}(z)+\beta B^{\prime}(z) s(t(z))\right|
$$

for $|z|=1$ and $|\beta|<1$. Now, using the continuity in zeros and $\beta$, we can obtain the (2.3), when some zeros of $s(t(z))$ lie on the unit circle $|z|=1$ and $|\beta| \leq 1$.

Applying Lemma 2.3 to the rational function $r(t(z))$ and $B(z) \sup _{|z|=1}|r(t(z))|$, we get the following.

Lemma 2.4. If $r(t(z)) \in \mathcal{R}_{m n}$, then for all $\beta \in \mathbb{C}$, with $|\beta| \leq 1$ and $|z|=1$, we have

$$
\left|B(z) r^{\prime}(t(z)) t^{\prime}(z)+\frac{\beta}{2} B^{\prime}(z) r(t(z))\right| \leq|B(z)|\left|1+\frac{\beta}{2}\right| \sup _{|z|=1}|r(t(z))| .
$$

Lemma 2.5. If $P(z)$ is a polynomial of degree $n$ having all zeros in $|z| \leq 1$, then

$$
\inf _{|z|=1}\left|P^{\prime}(z)\right| \geq n \inf _{|z|=1}|P(z)| \text {. }
$$


The result is best possible and equality in (2.4) holds for polynomials, having all zeros at the origin.

The above lemma is due to Aziz and Dawood [1].

\section{Main Results}

In this note, we shall use a parameter $\beta$ and obtain generalizations of (1.5), (1.6) and (1.7). We shall always assume that all the poles of $r(t(z)) \in \mathcal{R}_{m n}$ lie in $|z|>1$.

Theorem 3.1. If $r(t(z)) \in \mathcal{R}_{m n}$ and $|z|=1$, then for every $\beta$, with $|\beta| \leq 1$,

$$
\left|B(z) r^{\prime}(t(z)) t^{\prime}(z)+\frac{\beta}{2} B^{\prime}(z) r(t(z))\right|+\left|B(z)\left[(r(t(z)))^{*}\right]^{\prime}+\frac{\beta}{2} B^{\prime}(z)(r(t(z)))^{*}\right|
$$

$$
\leq\left|B^{\prime}(z)\right|\left\{\left|1+\frac{\beta}{2}\right|+\left|\frac{\beta}{2}\right|\right\} \sup _{|z|=1}|r(t(z))| .
$$

Proof. Let $M:=\sup _{|z|=1}|r(t(z))|$. Therefore, for every $\lambda \in \mathbb{C}$, with $|\lambda|>1$, $|r(t(z))|<|\lambda M B(z)|$ for $|z|=1$.

By Rouche's theorem, all the $m n$ zeros of $G(z)=r(t(z))+\lambda M B(z)$ lie in $|z|<1$. If $H(z)=B(z) \overline{G\left(\frac{1}{z}\right)}$, then $|H(z)|=|G(z)|$ for $|z|=1$ and hence, for any $\gamma$, with $|\gamma|<1$, the rational function $\gamma H(z)+G(z)$ has all $m n$ zeros in $|z|<1$. By applying Lemma 2.1 to $\gamma H(z)+G(z)$, we have

$$
2\left|B(z)\left(\gamma H^{\prime}(z)+G^{\prime}(z)\right)\right| \geq\left|B^{\prime}(z)\right||\gamma H(z)+G(z)|, \quad \text { for }|z|=1 .
$$

Since $B^{\prime}(z) \neq 0$ therefore, the right hand side of (3.2) is non zero. Thus, by using (i) of Lemma 2.2, we have for all $\beta \in \mathbb{C}$, with $|\beta|<1$,

$$
2 B(z)\left(\gamma H^{\prime}(z)+G^{\prime}(z)\right) \neq-\beta B^{\prime}(z)(\gamma H(z)+G(z)), \quad \text { for }|z|=1 .
$$

Equivalently, for $|z|=1$,

$$
-\gamma\left(2 B(z) H^{\prime}(z)+\beta B^{\prime}(z) H(z)\right) \neq-\left(2 B(z) G^{\prime}(z)+\beta B^{\prime}(z) G(z)\right),
$$

for $|\gamma|<1,|\beta|<1$. Using (ii) of Lemma 2.2 in (3.3), we have

$$
\left|2 B(z) G^{\prime}(z)+\beta B^{\prime}(z) G(z)\right| \leq\left|2 B(z) H^{\prime}(z)+\beta B^{\prime}(z) H(z)\right|,
$$

for $|z|=1,|\beta|<1$. Now, using $G(z)=r(t(z))+\lambda M B(z)$ and since

$$
\left.H(z)=B(z) \overline{G\left(\frac{1}{\bar{z}}\right)}=B(z) \overline{\left(r\left(t\left(\frac{1}{\bar{z}}\right)\right)\right.}+\bar{\lambda} M \overline{B\left(\frac{1}{\bar{z}}\right)}\right)=(r(t(z)))^{*}+\bar{\lambda} M,
$$

for $|z|=1$ in (3.4), we get, for $|\beta|<1$ and $|z|=1$,

$$
\begin{aligned}
& \left|2 B(z)\left[(r(t(z)))^{*}\right]^{\prime}+\beta B^{\prime}(z)(r(t(z)))^{*}+\bar{\lambda} \beta M B^{\prime}(z)\right| \\
\leq & \left|2 B(z) r^{\prime}(t(z)) t^{\prime}(z)+\beta B^{\prime}(z) r(t(z))+\lambda B(z) B^{\prime}(z)(2+\beta) M\right| .
\end{aligned}
$$


By choosing a suitable argument of $\lambda$ and applying Lemma 2.4 on the right hand side of (3.5), we get, for $|z|=1$ and $|\beta|<1$,

$$
\begin{aligned}
& \left|2 B(z)\left[(r(t(z)))^{*}\right]^{\prime}+\beta B^{\prime}(z)(r(t(z)))^{*}\right|-|\lambda|\left|\beta B^{\prime}(z)\right| M \\
\leq & |\lambda|\left|B(z) B^{\prime}(z)(2+\beta)\right| M-\left|2 B(z) r^{\prime}(t(z)) t^{\prime}(z)+\beta B^{\prime}(z) r(t(z))\right| .
\end{aligned}
$$

Note that $|B(z)|=1$ for $|z|=1$. Making $|\lambda| \rightarrow 1$ and using continuity for $|\beta|=1$ in (3.6), we get (3.1) and this proves the desired result.

For $t(z)=z$, Theorem 3.1 reduces to the following result.

Corollary 3.1. If $r \in \mathcal{R}_{n}$ and $|z|=1$, then for every $\beta$, with $|\beta| \leq 1$,

$$
\begin{aligned}
& \left|B(z) r^{\prime}(z)+\frac{\beta}{2} B^{\prime}(z) r(z)\right|+\left|B(z)\left(r^{*}(z)\right)^{\prime}+\frac{\beta}{2} B^{\prime}(z) r^{*}(z)\right| \\
\leq & \left|B^{\prime}(z)\right|\left\{\left|1+\frac{\beta}{2}\right|+\left|\frac{\beta}{2}\right|\right\} \sup _{|z|=1}|r(z)| .
\end{aligned}
$$

Remark 3.1. For $\beta=0$, (3.7) reduces to (1.5).

Theorem 3.2. If $r(t(z)) \in \mathcal{R}_{m n}$ is self-inversive and $|z|=1$, then for every $\beta$ with $|\beta| \leq 1$, we have

$$
\left|B(z) r^{\prime}(t(z)) t^{\prime}(z)+\frac{\beta}{2} B^{\prime}(z) r(t(z))\right| \leq \frac{\left|B^{\prime}(z)\right|}{2}\left\{\left|1+\frac{\beta}{2}\right|+\left|\frac{\beta}{2}\right|\right\} \sup _{|z|=1}|r(t(z))| .
$$

Proof. Since $r(t(z))$ is self-inversive, therefore, we have $(r(t(z)))^{*}=\lambda r(t(z))$ with $|\lambda|=1$. Hence, for all $\beta \in \mathbb{C}$,

$$
\left|B(z) r^{\prime}(t(z)) t^{\prime}(z)+\frac{\beta}{2} B^{\prime}(z) r(t(z))\right|=\left|B(z)\left[(r(t(z)))^{*}\right]^{\prime}+\frac{\beta}{2} B^{\prime}(z)(r(t(z)))^{*}\right| .
$$

Combining Theorem 3.1 and (3.9), we have for every $\beta$, with $|\beta| \leq 1$ and $|z|=1$,

$$
\begin{aligned}
2\left|B(z) r^{\prime}(t(z)) t^{\prime}(z)+\frac{\beta}{2} B^{\prime}(z) r(t(z))\right|= & \left|B^{\prime}(z) r^{\prime}(t(z)) t^{\prime}(z)+\frac{\beta}{2} B^{\prime}(z) r(t(z))\right| \\
& +\left|B(z)\left[(r(t(z)))^{*}\right]^{\prime}+\frac{\beta}{2} B^{\prime}(z)(r(t(z)))^{*}\right| \\
& \leq\left|B^{\prime}(z)\right|\left\{\left|1+\frac{\beta}{2}\right|+\left|\frac{\beta}{2}\right|\right\} \sup _{|z|=1}|r(t(z))|,
\end{aligned}
$$

which proves Theorem 3.2 completely.

Remark 3.2. If we take $\beta=0$ in inequality (3.8) and make use of the Lemma 2.5, after supposing that $t(z)$ has all its zeros in $|z| \leq 1$, we get the following result. 
Corollary 3.2. If $r(t(z)) \in \mathcal{R}_{m n}$ is self-inversive, where $t(z)$ has all its zeros in $|z| \leq 1$, then for $|z|=1$,

$$
\left|r^{\prime}(t(z))\right| \leq \frac{\left|B^{\prime}(z)\right|}{2 m \mu} \sup _{|z|=1}|r(t(z))|
$$

where $\mu=\inf _{|z|=1}|t(z)|$.

Remark 3.3. For $t(z)=z,(3.10)$ reduces to (1.6).

We end this section by proving the following interesting generalization of (1.7).

Theorem 3.3. Suppose $r(t(z)) \in \mathcal{R}_{m n}$ and all the mn zeros of $r(t(z))$ lie in $|z| \geq 1$. Then for every $\beta$, with $|\beta| \leq 1$ and $|z|=1$, we have

$$
\left|B(z) r^{\prime}(t(z)) t^{\prime}(z)+\frac{\beta}{2} B^{\prime}(z) r(t(z))\right| \leq \frac{\left|B^{\prime}(z)\right|}{2}\left\{\left|1+\frac{\beta}{2}\right|+\left|\frac{\beta}{2}\right|\right\} \sup _{|z|=1}|r(t(z))| .
$$

Proof. Since $r(t(z)) \in \mathcal{R}_{m n}$ has all its $m n$ zeros in $|z| \geq 1$ and $(r(t(z)))^{*}=$ $B(z) \overline{r\left(t\left(\frac{1}{\bar{z}}\right)\right)}$, therefore, all the zeros of $(r(t(z)))^{*}$ lie in $|z| \leq 1$. Also, $|r(t(z))|=$ $\left|(r(t(z)))^{*}\right|$ for $|z|=1$. Hence, by Lemma 2.3 , it follows for every $\beta$, with $|\beta| \leq 1$ and $|z|=1$,

$$
\left|B(z) r^{\prime}(t(z)) t^{\prime}(z)+\frac{\beta}{2} B^{\prime}(z) r(t(z))\right| \leq\left|B(z)\left[(r(t(z)))^{*}\right]^{\prime}+\frac{\beta}{2} B^{\prime}(z)(r(t(z)))^{*}\right| .
$$

Combining Theorem 3.1 and (3.12), we have for every $\beta$, with $|\beta| \leq 1$ and $|z|=1$,

$$
\begin{aligned}
2\left|B(z) r^{\prime}(t(z)) t^{\prime}(z)+\frac{\beta}{2} B^{\prime}(z) r(t(z))\right| \leq & \left|B^{\prime}(z) r^{\prime}(t(z)) t^{\prime}(z)+\frac{\beta}{2} B^{\prime}(z) r(t(z))\right| \\
& +\left|B(z)\left[(r(t(z)))^{*}\right]^{\prime}+\frac{\beta}{2} B^{\prime}(z)(r(t(z)))^{*}\right| \\
& \leq\left|B^{\prime}(z)\right|\left\{\left|1+\frac{\beta}{2}\right|+\left|\frac{\beta}{2}\right|\right\} \sup _{|z|=1}|r(t(z))|,
\end{aligned}
$$

which is equivalent to (3.11) and this completes the proof of Theorem 3.3.

Remark 3.4. If we take $\beta=0$ in (3.11) and assume that $t(z)$ has all its zeros in $|z| \leq 1$, we get (1.7) by virtue of Lemma 2.5.

Acknowledgements. The author is extremely grateful to the anonymous referee for many commendable suggestions and comments. 


\section{REFERENCES}

[1] A. Aziz and Q. M. Dawood, Inequalities for a polynomial and its derivative, J. Approx. Theory 54 (1988), 306-313.

[2] S. Bernstein, Sur l'ordre de la meilleure approximation des fonctions continues par des polynômes de degré donné, Mem. Cl. Sci. Acad. Roy. Belg. 4 (1912), 1-103.

[3] P. Borwein and T. Erdélyi, Polynomials and Polynomial Inequalities, Springer-Verlag, New York, 1995.

[4] M. I. Ganzburg, Sharp constants in V. A. Markov-Bernstein type inequalities of different metrics, J. Approx. Theory 215 (2017), 92-105.

[5] M. I. Ganzburg and S. Y. Tikhonov, On sharp constants in Bernstein-Nikolskii inequalities, Constr. Approx. 45 (2017), 449-466.

[6] I. Qasim and A. Liman, Bernstein type inequalities for rational functions, Indian J. Pure Appl. Math. 46 (2015), 337-348.

[7] S. Kalmykov and B. Nagy, Higher Markov and Bernstein inequalities and fast decreasing polynomials with prescribed zeros, J. Approx. Theory 226 (2018), 34-59.

[8] S. Kalmykov, B. Nagy and V. Totik, Bernstein- and Markov-type inequalities for rational functions, Acta Math. 219 (2017), 21-63.

[9] P. D. Lax, Proof of a conjecture of P. Erdös on the derivative of a polynomial, Bull. Amer. Math. Soc. 50 (1944), 509-513.

[10] M. A. Malik, On the derivative of a polynomial, J. Lond. Math. Soc. 1 (1969), 57-60.

[11] G. V. Milovanović, D. S. Mitrinović and Th. M. Rassias, Topics in Polynomials: Extremal Problems, Inequalities, Zeros, World Scientific, Singapore, 1994.

[12] P. Turán, Über die Ableitung von Polynomen, Compos. Math. 7 (1939), 89-95.

[13] Xin Li, A comparison inequality for rational functions, Proc. Amer. Math. Soc. 139 (2011), 1659-1665.

[14] X. Li, R. N. Mohapatra and R. S. Rodriguez, Bernstein-type inequalities for rational functions with prescribed poles, J. Lond. Math. Soc. 51 (1995), 523-531.

${ }^{1}$ Department of MAThematics,

UNIVERSITY OF KASHMIR,

HAZRATBAL SRINAGAR, INDIA

Email address: mabdullah_mir@yahoo.co.in 